\title{
STRONG ASYMPTOTICS ON THE SUPPORT OF THE MEASURE OF ORTHOGONALITY FOR POLYNOMIALS ORTHOGONAL WITH RESPECT TO A DISCRETE SOBOLEV INNER PRODUCT
}

\author{
Ana Foulquié Moreno and Francisco Marcellán
}

\begin{abstract}
In $[6,8]$ relative asymptotics for Sobolev orthogonal polynomials were studied. When the continuous part of the measure satisfies the Szegö condition, the strong asymptotics outside the support of the measure follow from $[6,8]$. In this paper, we analyze this problem on the support of the measure.
\end{abstract}

\section{Introduction and statements of results}

Some problems arising from approximation theory, mathematical physics, and number theory have motivated different generalizations of the standard notion of orthogonality. If $\mu$ is a finite positive Borel measure supported on the real line, then $\left\{p_{n}\right\}$ is the sequence of standard orthonormal polynomials (OP). with respect to $\mu$ when

$$
\int p_{n}(x) p_{m}(x) d \mu(x)= \begin{cases}0 & n \neq m \\ 1 & n=m\end{cases}
$$

and $p_{n}(x)=\alpha_{n} x^{n}+$ lower degree terms with $\alpha_{n}>0$ for $n \in \mathbb{N}$.

Some of these generalizations are the so-called Hermite-Padé polynomials, [3, 10], matrix polynomials, $[2,4,5]$, and orthogonal polynomials with respect to a linear homogeneous differential operator (OPDO) [1]. This last notion is important because we can apply the analytical methods used in the theory of Hermite-Padé approximants to OPDO. An important class among these notions are the Sobolev orthogonal polynomials (SOP) that are included in the class of OPDO. In this paper, we deal with the case of SOP with respect to a discrete Sobolev inner product $[6,8]$.

Definition 1. Let $\mu$ be a finite positive Borel measure, whose support $\mathcal{S}_{\mu}$ contains an infinite set of real points $\left(S_{\mu} \subset \mathbb{R}\right)$. A discrete Sobolev inner product is given by

$$
\langle h, g\rangle=\int h(x) g(x) d \mu(x)+\sum_{j=1}^{m} \sum_{i=0}^{N_{j}} M_{j, i} h^{(i)}\left(c_{j}\right) g^{(i)}\left(c_{j}\right)
$$

where $c_{j} \in \mathbb{R}, M_{j, i} \geq 0, m, N_{j}>0$.

Received February 23, 1996, revised September 20, 1996.

1991 Mathematics Subject Classification: Primary 33C45.

Key words and phrases: Sobolev orthogonal polynomials, asymptotic analysis, Szegő measures. 
In [6], a generalization of the above discrete Sobolev inner product in the following sense was considered

$$
\langle h, g\rangle=\int h(x) g(x) d \mu(x)+\sum_{j=1}^{m} \sum_{i=0}^{N_{j}} h^{(i)}\left(c_{j}\right) \mathcal{L}_{j, i}\left(g ; c_{j}\right)
$$

where $\mathcal{L}_{j, i}\left(g ; c_{j}\right)$ is the evaluation at $c_{j} \in \mathbb{C}$ of a linear differential operator with constant coefficients $\mathcal{L}_{j, i}$ acting on $g$ and $\mathcal{L}_{j, N_{j}} \neq 0, j=1, \ldots, m$. The motivation of this definition comes from the following orthogonality relation

$$
0=\int p(x) Q_{n}(x) d \mu(x)+\left.\sum_{j=1}^{m} \sum_{i=0}^{N_{j}} A_{j, i}\left(p(z) Q_{n}(z)\right)^{(i)}\right|_{z=c_{j}}, \quad p \in \mathbb{P}_{n-1},
$$

where $A_{j, i}$ may be complex numbers. The polynomials of the sequence $\left\{Q_{n}\right\}_{n \geq 0}$ are the denominators of the main diagonal sequence of the Padé approximants of Stieltjestype meromorphic functions

$$
f(z)=\int \frac{d \mu(x)}{z-x}+\sum_{j=1}^{m} \sum_{i=0}^{N_{j}} A_{j, i} \frac{i !}{\left(z-c_{j}\right)^{i+1}}, \quad A_{j, N_{j}} \neq 0 .
$$

Given $j=1, \ldots, m$, let $J_{j}$ be the maximum order of the differential operators $\mathcal{L}_{j, i}$, $i=0, \ldots, N_{j}$. Then

$$
\mathcal{L}_{j, i}(g ; x)=\sum_{k=0}^{J_{j}} \gamma_{i, k}^{j} g^{(k)}(x) .
$$

For a fixed $j$, let $\mu_{j}=\left(\gamma_{i, k}^{j}\right), i=0, \ldots, N_{j}, k=0, \ldots, J_{j}$, be the matrix whose elements are the coefficients of the operator $\mathcal{L}_{j, i}$. Denote by $\mu_{j}{ }^{*}$ the matrix obtained from $\mu_{j}$, deleting all the zero rows and columns. Following [6], we say that (1) is regular if, for each $j=1, \ldots, m$, the matrix $\mu_{j}^{*}$ is a square matrix with non-zero determinant. We denote the dimension of $\mu_{j}^{*}$ by $I_{j}$, and $I=\sum_{j=1}^{m} I_{j}$.

The aim of our paper is the analysis of the strong asymptotics for the polynomials orthogonal with respect to (1) in the regular case when $\left\{c_{j}\right\} \in \mathbb{C} \backslash \mathcal{S}_{\mu}, j=1, \ldots, m$. So we denote by $S_{n}, n \in \mathbb{Z}_{+}$, the monic polynomial of least degree such that

$$
\left\langle p, S_{n}\right\rangle=0 \quad p \in \mathbb{P}_{n-1} .
$$

If the inner product is positive definite, then $\operatorname{deg} S_{n}$ is $n$, and thus all the $S_{n}$ 's are distinct. In general, this is not true, and for different values of $n$, we can have the same $S_{n}$.

Despite an extensive effort to investigate the properties of SOP, not much progress has been attained regarding their asymptotics for general conditions on the inner products. The first essential contribution to this topic was [6]. There a new class of measures was introduced.

Definition 2. Let $\mu$ be a complex measure. We say that $\mu \in M_{\mathbb{C}}(0,1)$ if the corresponding orthonormal polynomials $\left\{q_{n}\right\}$ satisfy

$$
x q_{n}(x)=\alpha_{n+1} q_{n+1}(x)+\beta_{n} q_{n}(x)+\alpha_{n} q_{n-1}(x), \quad n \geq n_{0}, \quad \alpha_{n}, \beta_{n} \in \mathbb{C}
$$


with

$$
\begin{gathered}
\lim _{n \rightarrow \infty} \alpha_{n}=\frac{1}{2}, \quad \lim _{n \rightarrow \infty} \beta_{n}=0, \\
\int\left|q_{n}(x) q_{n+m}(x)\right||d \mu(x)| \leq C<\infty
\end{gathered}
$$

where the support of $\mu$ is equal to $[-1,1] \cup E$, and $E$ is at most a denumerable set of isolated points in $\mathbb{C} \backslash[-1,1]$, with $E^{\prime} \subset[-1,1]$.

The following asymptotic behavior for the sequence $\left\{q_{n}\right\}$ holds

$$
\frac{q_{n+1}^{(\nu)}(z)}{q_{n}^{(\nu)}(z)} \rightrightarrows \varphi(z), \quad K \subset \mathbb{C} \backslash S_{\mu}
$$

where

$$
\varphi(z)=z+\sqrt{z^{2}-1}, \quad|\varphi(z)|>1
$$

and

$$
\frac{1}{n} \frac{q_{n}^{(\nu+1)}(z)}{q_{n}^{(\nu)}(z)} \rightrightarrows \frac{1}{\sqrt{z^{2}-1}}, \quad K \subset \mathbb{C} \backslash S_{\mu} .
$$

Notation. The symbol $\rightrightarrows$ means, in this work, uniform convergence on compact subsets of the indicated region.

For this class of measures, a method for the analysis of relative asymptotic properties of SOP with respect to OP has been developed. In particular, the following theorem was proved [6, Th. 4].

Theorem 3. Consider a regular inner product of type (1) such that $\mu \in M_{\mathbb{C}}(0,1)$ and $c_{1}, \ldots, c_{m} \in \mathbb{C} \backslash S_{\mu}$. Let $\left\{L_{n}\right\}, n \in \mathbb{Z}_{+}$be the sequence of monic orthogonal polynomials with respect to $\mu$, and let $\left\{S_{n}\right\}, n \in \mathbb{Z}_{+}$be the monic orthogonal polynomials with respect to the above inner product. Then for all sufficiently large $n, \operatorname{deg} S_{n}$ is $n$ and each point $c_{j}$ attracts exactly $I_{j}$ zeros of $S_{n}$, while the other zeros concentrate on $S_{\mu}$. Also for each fixed $\nu \in \mathbb{Z}_{+}$,

$$
\frac{S_{n}{ }^{(\nu)}(z)}{L_{n}{ }^{(\nu)}(z)} \rightrightarrows \prod_{j=1}^{m}\left(\frac{\left(\varphi(z)-\varphi\left(c_{j}\right)\right)^{2}}{2 \varphi(z)\left(z-c_{j}\right)}\right)^{I_{j}}, \quad K \subset \overline{\mathbb{C}} \backslash S_{\mu}
$$

We use the following corollary [6, Cor.2] later.

Corollary 4. Under the hypothesis of the above theorem, for all sufficiently large $n$, $\left\langle S_{n}, S_{n}\right\rangle \neq 0$. Let $\eta_{n}$ be the leading coefficient of $l_{n}$, the $n$-th orthonormal polynomial with respect to $\mu$. Then $\gamma_{n}=\left\langle S_{n}, S_{n}\right\rangle^{-\frac{1}{2}}$ may be taken so that

$$
\lim _{n \rightarrow \infty} \frac{\gamma_{n}}{\eta_{n}}=\prod_{j=1}^{m} \frac{1}{\varphi\left(c_{j}\right)^{I_{j}}}
$$

and, in particular,

$$
\lim _{n \rightarrow \infty} \frac{\gamma_{n+1}}{\gamma_{n}}=2
$$


The advantage of such a theorem is the following. If we know the asymptotic behavior of the OP, then we can deduce the corresponding behavior for the SOP. To illustrate this with an example, we can consider $d \mu(x)=\omega(x) d x$ where $\omega(x)$ is a positive and integrable function in $[-1,1]$ satisfying the Szegö condition

$$
\int_{-1}^{1} \frac{\ln \omega(x)}{\sqrt{1-x^{2}}} d x>-\infty
$$

We denote by $\left\{l_{n}=\eta_{n} x^{n}+\cdots\right\}$ the set of polynomials orthonormal with respect to the function $\omega(x)$

$$
\int_{-1}^{1} l_{n}(x) l_{m}(x) \omega(x) d x=\left\{\begin{array}{ll}
0 & n \neq m \\
1 & n=m
\end{array} .\right.
$$

It is well known [11] that the function

$$
D_{\omega}(z)=\exp \left\{\frac{1}{4 \pi} \int_{-\pi}^{+\pi} \frac{1+z e^{-i t}}{1-z e^{-i t}} \ln (\omega(\cos t)|\sin t|) d t\right\}
$$

satisfies

(a) $D_{\omega}(z) \in H_{2}$,

(b) $D_{\omega}\left(e^{i \theta}\right)=\lim _{r \rightarrow 1^{-}} D_{\omega}\left(r e^{i \theta}\right)$ exists a.e. and $\left|D_{\omega}\left(e^{i \theta}\right)\right|^{2}=\omega(\cos \theta)|\sin \theta|$,

(c) $D_{\omega}(z) \neq 0,|z|<1$, and $D_{\omega}(0)>0$.

We have the following strong asymptotics for $\left\{l_{n}\right\}$ (see [11], Theorems 12.1.2 and 12.1.4):

(a) Outside the support:

$$
l_{n}\left(\frac{1}{2}\left(z+\frac{1}{z}\right)\right)=\frac{1}{\sqrt{2 \pi}} \frac{1}{z^{n}} \frac{1}{D_{\omega}(z)}[1+o(1)], \quad|z|<1 .
$$

(b) On the support:

$$
l_{n}(\cos \theta)=\frac{1}{\sqrt{2 \pi}}\left[\frac{1}{D_{\omega}\left(e^{i \theta}\right)} e^{-i n \theta}+\frac{1}{\overline{D_{\omega}\left(e^{i \theta}\right)}} e^{i n \theta}\right]+o(1)
$$

where $o(1)$ may be understood in the sense of convergence in $\mathcal{L}_{2, \omega}$.

(c) The leading coefficient:

$$
\eta_{n}=\frac{1}{\sqrt{2 \pi}} 2^{n} \frac{1}{D_{\omega}(0)}[1+o(1)]
$$

Combining these results with [6, Th. 4], we obtain as an immediate consequence the strong asymptotics of SOP outside the support of the measure of orthogonality.

Corollary 5. Using Corollary 4, we can define for sufficiently large $n, s_{n}=\gamma_{n} S_{n}$. Then, for $|z|<1$

$$
s_{n}\left(\frac{1}{2}\left(z+\frac{1}{z}\right)\right)=\frac{1}{\sqrt{2 \pi}} \frac{1}{z^{n}} \frac{\prod_{j=1}^{m} \beta_{j}(z)^{I_{j}}}{D_{\omega}(z)}[1+o(1)]
$$


uniformly for $z \in K \subset\{z:|z|<1\} \backslash\left\{z_{j}\right\}_{j=1}^{m}$, where we have used the notation

$$
\begin{gathered}
\beta_{j}(z):=\frac{z_{j}-z}{\left(1-z z_{j}\right)}, \\
\varphi(x)=x+\sqrt{x^{2}-1}, \quad|\varphi(x)|>1, \\
\frac{1}{2}(z+1 / z)=x, \quad \varphi(x)=\frac{1}{z}, \\
\frac{1}{2}\left(z_{j}+1 / z_{j}\right)=c_{j}, \quad \varphi\left(c_{j}\right)=\frac{1}{z_{j}} .
\end{gathered}
$$

At the same time, we should mention that the theorem from [6, Th. 4] does not allow us to make a conclusion about asymptotics of SOP on the support of the measure of orthogonality. This is not a surprise because the values of the entries of the matrix $\mu_{j}$ do not appear in [6, Th. 4], but as we show later, the asymptotics at the mass points strictly depend on them. So an investigation of the strong asymptotic properties of the polynomials on the support of the measure requires a more delicate technique to be developed. The main aim of the present paper is to fill this gap. We prove the following.

Theorem 6. Let $\omega$ be a positive and integrable function which satisfies the Szegö condition $\int_{-1}^{1} \ln \omega(x) / \sqrt{1-x^{2}} d x>-\infty$. Let us consider an inner product (1) in the regular case and $\left\{c_{j}\right\}_{j=1}^{m} \in \mathbb{C} \backslash \mathcal{S}_{\mu}$ where $d \mu(x)=\omega(x) d x$. For sufficiently large $N \in \mathbb{N}$, let $\left\{S_{n}=x^{n}+\cdots\right\}_{n \geq N}$ be the sequence of monic orthogonal polynomials with respect to (1), and $\gamma_{n}=\left\langle S_{n}, S_{n}\right\rangle^{-1 / 2}$. Let $s_{n}=\gamma_{n} S_{n}$. Then we have the following asymptotics for $\left\{s_{n}\right\}_{n \geq N}$ :

(a) On the interval $[-1,1]$,

$$
\left\|s_{n}(\cos \theta)-\frac{1}{\sqrt{2 \pi}}\left[\frac{1}{D_{\omega}\left(e^{i \theta}\right) / \beta\left(e^{i \theta}\right)} e^{-i n \theta}+\frac{1}{\left[\overline{D_{\omega}\left(e^{i \theta}\right)} / \beta\left(e^{-i \theta}\right)\right]} e^{i n \theta}\right]\right\|=o(1)
$$

where $\|\cdot\|$ means the norm in $\mathcal{L}^{2}(\omega(\cos \theta)|\sin \theta| d \theta,[-\pi, \pi])$ and

$$
\beta(z):=\prod_{j=1}^{m}\left[\frac{z_{j}-z}{\left(1-z z_{j}\right)}\right]^{I_{j}} \text {. }
$$

(b) At the mass points $\left\{c_{j}\right\}_{j=1}^{m}$, in the case that $J_{j}=N_{j}=I_{j}-1$,

where

$$
\left(\begin{array}{c}
s_{n}\left(c_{j}\right) \\
s_{n}^{\prime}\left(c_{j}\right) \\
\vdots \\
s_{n}^{\left(J_{j}\right)}\left(c_{j}\right)
\end{array}\right)=\left(\mu_{j}\right)^{-1}\left(G_{j}\right)^{-1}\left(\begin{array}{c}
h_{n}\left(c_{j}\right) \\
h_{n}^{\prime}\left(c_{j}\right) \\
\vdots \\
\frac{1}{J_{j} !} h_{n}^{\left(J_{j}\right)}\left(c_{j}\right)
\end{array}\right)[1+o(1)]
$$

$$
\begin{gathered}
h_{n}(x):=\tilde{h}_{n}\left(\frac{1}{\varphi(x)}\right), \quad \varphi(x)=x+\sqrt{x^{2}-1} \\
\tilde{h}_{n}(z):=-\frac{\sqrt{2 \pi}}{2^{M-1}} D_{\omega}(z) \frac{1}{z-1 / z} z^{n-M} \prod_{k=1}^{m} \frac{\left(z z_{k}-1\right)^{2\left(J_{k}+1\right)}}{z_{k}^{J_{k}+1}}
\end{gathered}
$$


and $G_{j}, j=1, \ldots, m$, are the following matrices:

$$
G_{j}(k, l)= \begin{cases}0 & J_{j}+2-k>l, \\
\left(\begin{array}{c}
l-1 \\
J_{j}+1-k
\end{array}\right)\left(J_{j}+1-k\right) ! g_{j}^{\left(l-\left(J_{j}+2-k\right)\right)}\left(c_{j}\right) & J_{j}+2-k \leq l,\end{cases}
$$

and $g_{j}(x)=\prod_{i=1, i \neq j}^{m}\left(x-c_{i}\right)^{J_{i}+1}$.

For a better understanding of the result, we give some corollaries.

Corollary 7. We first consider the simplest case $N_{j}=0$, and

$$
\langle h, g\rangle=\int_{-1}^{1} h(x) g(x) \omega(x) d x+\gamma h(c) g(c), \quad c \in \mathbb{R}, \quad \gamma>0 .
$$

In this case, if we denote $\frac{1}{2}\left(z_{1}+1 / z_{1}\right)=c$ with $\left|z_{1}\right|<1$, then

$$
\begin{gathered}
\gamma_{n}=\frac{2^{n}}{D_{\omega}(0)} \frac{\left|z_{1}\right|}{\sqrt{2 \pi}}[1+o(1)], \quad \gamma_{n}>0 \\
\gamma s_{n}(c)=\frac{\left|z_{1}\right|}{2} h_{n}\left(z_{1}\right)[1+o(1)] \\
h_{n}(z)=\sqrt{2 \pi} \frac{D_{\omega}\left(z_{1}\right)}{1 / 2} z_{1}^{n-1}\left(z_{1}-\frac{1}{z_{1}}\right) .
\end{gathered}
$$

Denoting $F(z)=\frac{D_{\omega}(z)\left(z z_{1}-1\right)\left|z_{1}\right|}{\left(z-z_{1}\right) z_{1}}$, we get that

$$
s_{n}(c)=\frac{D_{\omega}\left(z_{1}\right)}{\frac{1}{z_{1}-1 / z_{1}}}\left|z_{1}\right| \frac{\sqrt{2 \pi}}{\gamma} z_{1}^{n-1}[1+o(1)]=\frac{z_{1}^{n-1}}{\gamma} \sqrt{2 \pi} \operatorname{Res}_{z=z_{1}} F(z)[1+o(1)]
$$

holds.

This result agrees with [9, (17), p.2691].

Corollary 8. Now if we consider

$$
\langle h, g\rangle=\int_{-1}^{1} h(x) g(x) \omega(x) d x+\gamma_{00} h(c) g(c)+\gamma_{11} h^{\prime}(c) g^{\prime}(c), \quad c \in \mathbb{C} \backslash[-1,1],
$$

then in this case $F(z)=\frac{D_{\omega}(z)}{\left(z-z_{1}\right)^{2}}\left(z z_{1}-1\right)^{2}$, and

$$
\left(\begin{array}{c}
s_{n}(c) \\
s_{n}^{\prime}(c)
\end{array}\right)=\frac{\sqrt{2 \pi} z_{1}^{n}}{\gamma_{00} \gamma_{11}}\left(\begin{array}{cc}
0 & \gamma_{11} \\
\gamma_{00} & 0
\end{array}\right)\left(\begin{array}{c}
\frac{1}{2} \frac{z_{1}^{2}-1}{z_{1}^{3}} \rho_{1} \\
\frac{1}{z_{1}} \rho_{2}+\left[\frac{1}{z_{1}^{3}}(n-1)-\frac{2}{z_{1}^{2}-1}\right] \rho_{1}
\end{array}\right)[1+o(1)],
$$

where $\rho_{1}=\operatorname{Res}_{z=z_{1}}\left(\left(z-z_{1}\right) F(z)\right)$ and $\rho_{2}=\operatorname{Res}_{z=z_{1}} F(z)$.

The method of proof of Theorem 6 is based on the investigation of the coefficients of a polynomial modification of SOP in terms of OP

$$
w_{M}(x) S_{n}(x)=L_{n+M}(x)+\sum_{j=1}^{2 M} a_{n, j} L_{n+M-j}(x)
$$

where $w_{M}(x)=\prod_{j=1}^{m}\left(x-c_{j}\right)^{N_{j}+1}$ and $\operatorname{deg} w_{M}$ is equal to $M$.

As an intermediate step, which at the same time is of independent interest, we prove 
Lemma 9. Consider a regular inner product of type (1) such that $\mu \in M_{\mathbb{C}}(0,1)$ and $c_{1}, \ldots, c_{m} \in \mathbb{C} \backslash S_{\mu}$. For sufficiently large $n$, let $S_{n}(x)$ be the monic Sobolev orthogonal polynomial of degree $n$ with respect to this inner product. Let $L_{n}(x)=x^{n}+\cdots$ be the monic orthogonal polynomial of degree $n$ with respect to $d \mu(x)$. Then

$$
w_{M}(x) S_{n}(x)=L_{n+M}(x)+\sum_{j=1}^{2 M} a_{n, j} L_{n+M-j}(x)
$$

where $w_{M}(x)=\prod_{j=1}^{m}\left(x-c_{j}\right)^{N_{j}+1}, \operatorname{deg} w_{M}(x)=M$. Furthermore, $\lim _{n \rightarrow \infty} a_{n, j}$ exists for $1 \leq j \leq m$.

This theorem gives us an analog of condition (2) of $M_{\mathbb{C}}(0,1)$ in the sense that the limits of the coefficients in a recurrence relation exist. The special case in the above theorems when $J_{j}=N_{j}=0$ was proved by Nikishin [9]. The next section is devoted to the proofs of Lemma 9 and of Theorem 6.

\section{Proof of the main results}

Proof of Lemma 9. The proof is given in two steps.

1. We consider $p(z)=w_{M}(z) p_{1}(z)$ where $p_{1}$ is an arbitrary polynomial of degree at most $n-M-1$. We have

$$
0=\left\langle w_{M}(z) p_{1}(z), S_{n}(z)\right\rangle=\int p_{1}(z) w_{M}(z) S_{n}(z) d \mu(z) .
$$

By Corollary 5, we know that for $n \geq n_{0}$

$$
\int L_{n}^{2}(x) d \mu(x) \neq 0
$$

We can conclude that any polynomial, for sufficiently large $n$, satisfying (6), must be of degree at least $n-M$. If we try to write

$$
w_{M}(z) S_{n}(z)=L_{n+M}(z)+\sum_{j=1}^{2 M} a_{n, j} L_{n+M-j}(z),
$$

then to do this we require that

$$
p_{n}(z)=\frac{a_{n, 0} L_{n+M}(z)+\sum_{j=1}^{2 M} a_{n, j} L_{n+M-j}(z)}{w_{M}(z)}
$$

be a polynomial. For doing this, we have $M$ homogeneous linear equations in the unknowns $\left\{a_{n, j}\right\}, j=0, \ldots, 2 M$.

With the above condition, one has

$$
0=\left\langle w_{M}(z) p_{1}(z), p_{n}(z)\right\rangle \quad 0 \leq \operatorname{deg} p_{1} \leq n-M-1 .
$$

Now, if we also impose

$$
0=\left\langle z^{k}, p_{n}(z)\right\rangle \quad k=0, \ldots, M-1,
$$

then we can guarantee a non-trivial solution for (7) and (8) because we have $2 M$ homogeneous linear equations and $2 M+1$ unknowns. Thus this polynomial $p_{n}$ satisfies the same orthogonality condition as $S_{n}$ and, for sufficiently large $n, \operatorname{deg} S_{n}=n$ so $a_{n, 0} \neq 0$. Taking the normalization $a_{n, 0}=1$, we can say $p_{n}=S_{n}$ and (5) holds. 
2. To prove the existence of the limit of $a_{n, j}$ for $j=1, \ldots, 2 M$, we follow the technique used in [6]. Let

$$
w_{M}(z) S_{n}(z)=L_{n+M}(z)+\sum_{j=1}^{2 M} a_{n, j} \bar{L}_{n+M-j}(z)
$$

We consider

$$
\Omega_{n}(z)=\frac{w_{M}(z) S_{n}(z)}{L_{n-M}(z)} \quad \text { and } \quad a_{n}^{*}=\frac{1}{1+\sum_{j=1}^{2 M}\left|a_{n, j}\right|}
$$

Set

$$
\Omega_{n}^{*}=a_{n}^{*} \Omega_{n}=a_{n}^{*} \frac{L_{n+M}(z)}{L_{n-M}(z)}+\sum_{j=1}^{2 M} a_{n, j}^{*} \frac{L_{n+M-j}(z)}{L_{n-M}(z)}
$$

where $a_{n, j}^{*}=a_{n}^{*} a_{n, j}$. The new sequence $\left\{a_{n, j}^{*}\right\}$ is uniformly bounded. Let $\Lambda$ be a subset of $\mathbb{N}$ such that for $j=1, \ldots, 2 M$

$$
\lim _{n \rightarrow \infty, n \in \Lambda} a_{n, j}^{*}=a_{j}^{*} \quad \text { and } \quad \lim _{n \rightarrow \infty, n \in \Lambda} a_{n}^{*}=a_{0}^{*} .
$$

Taking the limit in (9), when $n \rightarrow \infty, n \in \Lambda$, and making use of (3) and (4),

$$
\begin{gathered}
a_{0}^{*} \prod_{j=1}^{m} \frac{\left(z-c_{j}\right)^{N_{j}+1-I_{j}}\left(\varphi(z)-\varphi\left(c_{j}\right)\right)^{2 I_{j}}}{(2 \varphi(z))^{I_{j}}}\left(\frac{\varphi(z)}{2}\right)^{M} \\
=a_{0}^{*}\left(\frac{\varphi(z)}{2}\right)^{2 M}+\sum_{j=1}^{2 M} a_{j}^{*}\left(\frac{\varphi(z)}{2}\right)^{2 M-j}
\end{gathered}
$$

for $z \in \mathbb{C} \backslash S_{\mu}$. If we notice that $\left|a_{n}^{*}\right|+\sum_{j=1}^{2 M}\left|a_{n, j}^{*}\right|=1$, then it is clear that $\left|a_{0}^{*}\right|+\sum_{j=1}^{2 M}\left|a_{j}^{*}\right|=1$.

Now if we use the fact that $\left\{\varphi^{k}\right\}$ for $k=0, \ldots, 2 M$ is a set of linearly independent functions because $\varphi$ is a one-to-one holomorphic function, then it holds that $a_{0}^{*} \neq 0$.

In particular, this implies that $\left\{a_{n, j}\right\}$ is uniformly bounded, so to prove the existence of the limits, we only need to prove the uniqueness of the limit points for every $j=1, \ldots, 2 M$.

Let $\Lambda$ be a subset of $\mathbb{N}$ such that for $j=1, \ldots, 2 M$, the $\operatorname{limits}_{\lim } \lim _{n \rightarrow \Lambda} a_{n, j}=a_{j}$ exist. Taking $\lim _{n \rightarrow \infty, n \in \Lambda} \Omega_{n}(z)$, one obtains

$$
\prod_{j=1}^{m} \frac{\left(z-c_{j}\right)^{N_{j}+1-I_{j}}\left(\varphi(z)-\varphi\left(c_{j}\right)\right)^{2 I_{j}}}{(2 \varphi(z))^{I_{j}}}\left(\frac{\varphi(z)}{2}\right)^{M}=\left(\frac{\varphi(z)}{2}\right)^{2 M}+\sum_{j=1}^{2 M} a_{j}\left(\frac{\varphi(z)}{2}\right)^{2 M-j}
$$

for $z \in \mathbb{C} \backslash S_{\mu}$.

From this last equation, we have the uniqueness of $a_{j}$.

Proof of Theorem 6. First we are going to deduce the strong asymptotics on $[-1,1]$. 
Let

$$
w_{M}(z)=\prod_{j=1}^{m}\left(z-c_{j}\right)^{N_{j}+1}
$$

We expand $S_{n} w_{M}$ in terms of $\left\{L_{n}\right\}$.

$$
S_{n}(z) w_{M}(z)=L_{n+M}(z)+\sum_{j=1}^{2 M} a_{n, j} L_{n+M-j}(z) .
$$

Dividing by $S_{n}$,

$$
\begin{aligned}
w_{M}(z) & =\frac{L_{n+M}(z)}{S_{n}(z)}+\sum_{j=1}^{2 M} a_{n, j} \frac{L_{n+M-j}(z)}{S_{n}(z)} \\
& =\frac{L_{n+M}(z)}{L_{n}(z)} \frac{L_{n}(z)}{S_{n}(z)}+\sum_{j=1}^{2 M} a_{n, j} \frac{L_{n+M-j}(z)}{L_{n}(z)} \frac{L_{n}(z)}{S_{n}(z)}
\end{aligned}
$$

Now taking the limit in a compact set off supp $\mu$, using (4) as well as the existence of $\lim a_{n, j}=a_{j}$, (Lemma 9)

$$
\begin{aligned}
& w_{M}(z)=\prod_{j=1}^{m}\left(\frac{2 \varphi(z)\left(z-c_{j}\right)}{\left(\varphi(z)-\varphi\left(c_{j}\right)\right)^{2}}\right)^{I_{j}}\left[\left(\frac{\varphi(z)}{2}\right)^{M}+\sum_{j=1}^{2 M} a_{j}\left(\frac{\varphi(z)}{2}\right)^{M-j}\right] \\
& \prod_{j=1}^{m}\left(\varphi(z)-\varphi\left(c_{j}\right)\right)^{2 I_{j}}\left(z-c_{j}\right)^{N_{j}+1-I_{j}} \\
&=2^{I} \varphi^{I}(z)\left[\left(\frac{\varphi(z)}{2}\right)^{M}+\sum_{j=1}^{2 M} a_{j}\left(\frac{\varphi(z)}{2}\right)^{M-j}\right]
\end{aligned}
$$

Now, denoting $\varphi(z)=t$, we arrive at the equation,

$$
\begin{aligned}
\prod_{j=1}^{m}\left(t-\varphi\left(c_{j}\right)\right)^{2 I_{j}} & \left(\frac{1}{2}\left(t+\frac{1}{t}\right)-c_{j}\right)^{N_{j}+1-I_{j}} \\
= & 2^{I} t^{I}\left[\left(\frac{t}{2}\right)^{M}+\sum_{j=1}^{2 M} a_{j}\left(\frac{t}{2}\right)^{M-j}\right] .
\end{aligned}
$$

that these $a_{j}, j=1, \ldots, 2 M$ verify. We come back to (10), and, putting $z=\cos \theta$, we have

$$
S_{n}(\cos \theta) w_{M}(\cos \theta)=L_{n+M}(\cos \theta)+\sum_{j=1}^{2 M} a_{n, j} L_{n+M-j}(\cos \theta)
$$

Now using the strong asymptotics for $L_{n}$ on $[-1,1]$, we deduce 


\section{$S_{n}(\cos \theta) w_{M}(\cos \theta)$}

$$
\begin{aligned}
= & \frac{\frac{1}{\sqrt{2 \pi}}\left[\frac{1}{D_{\omega}\left(e^{i \theta}\right)} e^{-i(n+M) \theta}+\frac{1}{\overline{D_{\omega}\left(e^{i \theta}\right)}} e^{i(n+M) \theta}\right]}{\frac{1}{\sqrt{2 \pi}} 2^{n+M} \frac{1}{D_{\omega}(0)}} \\
& +\sum_{j=1}^{2 M} a_{j} \frac{D_{\omega}(0)}{2^{n+M-j}}\left[\frac{1}{D_{\omega}\left(e^{i \theta}\right)} e^{-i(n+M-j) \theta}+\frac{1}{\overline{D_{\omega}\left(e^{i \theta}\right)}} e^{i(n+M-j) \theta}\right]+o(1) \\
= & \frac{D_{\omega}(0)}{2^{n+M}}\left[\frac{1}{D_{\omega}\left(e^{i \theta}\right)} e^{-i(n+M) \theta}+\frac{1}{\overline{D_{\omega}\left(e^{i \theta}\right)}} e^{i(n+M) \theta}\right] \\
& +\sum_{j=1}^{2 M} a_{j} \frac{D_{\omega}(0)}{2^{n+M-j}}\left[\frac{1}{D_{\omega}\left(e^{i \theta}\right)} e^{-i(n+M-j) \theta}+\frac{1}{\overline{D_{\omega}\left(e^{i \theta}\right)}} e^{i(n+M-j) \theta}\right]+o(1) \\
= & \frac{D_{\omega}(0)}{D_{\omega}\left(e^{i \theta}\right)}\left[\frac{e^{-i n \theta}}{2^{n}}\left[\frac{1}{2^{M}} e^{-i M \theta}+\sum_{j=1}^{2 M} a_{j} \frac{1}{2^{M-j}} e^{-i(M-j) \theta}\right]\right] \\
& +\frac{D_{\omega}(0)}{\overline{D_{\omega}\left(e^{i \theta}\right)}}\left[\frac{e^{i n \theta}}{2^{n}}\left[\frac{1}{2^{M}} e^{i M \theta}+\sum_{j=1}^{2 M} a_{j} \frac{1}{2^{M-j}} e^{i(M-j) \theta}\right]\right]+o(1) .
\end{aligned}
$$

Using the notation

$$
e^{i \theta}=z, \quad \frac{1}{\varphi\left(c_{j}\right)}=z_{j}, \quad \frac{1}{2}\left(z_{j}+\frac{1}{z_{j}}\right)=c_{j},
$$

we have, by (11),

$$
\begin{gathered}
S_{n}(\cos \theta)=\frac{\frac{D_{\omega}(0)}{D_{\omega}(z)}\left[\frac{1}{2^{n} z^{n}} \prod_{j=1}^{m}\left[\frac{1}{z}-\frac{1}{z_{j}}\right]^{2 I_{j}} \frac{z^{I}}{2^{I}}\right]}{\prod_{j=1}^{m}\left[\frac{1}{2}\left(z+\frac{1}{z}\right)-\frac{1}{2}\left(z_{j}+\frac{1}{z_{j}}\right)\right]^{I_{j}}} \\
+\frac{\frac{D_{\omega}(0)}{D_{\omega}(z)}\left[\frac{z^{n}}{2^{n}} \prod_{j=1}^{m}\left[z-\frac{1}{z_{j}}\right]^{2 I_{j}} \frac{1}{2^{I} z^{I}}\right]}{\prod_{j=1}^{m}\left[\frac{1}{2}\left(z+\frac{1}{z}\right)-\frac{1}{2}\left(z_{j}+\frac{1}{z_{j}}\right)\right]^{I_{j}}}+o(1) \\
=\frac{D_{\omega}(0)}{D_{\omega}(z)}\left[\frac{1}{2^{n} z^{n}} \prod_{j=1}^{m}\left[\frac{z_{j}-z}{z_{j}\left(1-z z_{j}\right)}\right]^{I_{j}}\right] \\
+\frac{D_{\omega}(0)}{D_{\omega}(z)}\left[\frac{z^{n}}{2^{n}} \prod_{j=1}^{m}\left[\frac{z z_{j}-1}{z_{j}\left(z-z_{j}\right)}\right]^{I_{j}}\right]+o(1) .
\end{gathered}
$$


If we denote

$$
\alpha(z)=\prod_{j=1}^{m}\left[\frac{z_{j}-z}{z_{j}\left(1-z z_{j}\right)}\right]^{I_{j}}
$$

then

$$
S_{n}(\cos \theta)=\frac{D_{\omega}(0)}{\frac{D_{\omega}\left(e^{i \theta}\right)}{\alpha\left(e^{i \theta}\right)}} \frac{1}{2^{n}} e^{-i n \theta}+\frac{D_{\omega}(0)}{\left[\frac{\overline{D_{\omega}\left(e^{i \theta}\right)}}{\alpha\left(e^{-i \theta}\right)}\right]} \frac{1}{2^{n}} e^{i n \theta}+o(1)
$$

For sufficiently large $n$ the leading coefficient of $s_{n}$ is denoted by $\gamma_{n}$. From Corollary 4

$$
\gamma_{n}=\frac{1}{\sqrt{2 \pi}} 2^{n} \frac{1}{D_{\omega}(0)} \frac{1}{\prod_{j=1}^{m} \varphi\left(c_{j}\right)^{I_{j}}}+o(1) \text { holds. }
$$

Using this and (12), we have

$$
s_{n}(\cos \theta)=\frac{1}{\sqrt{2 \pi}} \prod_{j=1}^{m} z_{j}^{I_{j}}\left[\frac{1}{\frac{D_{\omega}\left(e^{i \theta}\right)}{\alpha\left(e^{i \theta}\right)}} e^{-i n \theta}+\frac{1}{\left[\frac{\overline{D_{\omega}\left(e^{i \theta}\right)}}{\alpha\left(e^{-i \theta}\right)}\right]} e^{i n \theta}\right]+o(1) .
$$

If we denote

$$
\beta(z):=\prod_{j=1}^{m}\left[\frac{z_{j}-z}{\left(1-z z_{j}\right)}\right]^{I_{j}}
$$

then

$$
s_{n}(\cos \theta)=\frac{1}{\sqrt{2 \pi}}\left[\frac{1}{\frac{D_{\omega}\left(e^{i \theta}\right)}{\beta\left(e^{i \theta}\right)}} e^{-i n \theta}+\frac{1}{\left[\frac{\overline{D_{\omega}\left(e^{i \theta}\right)}}{\beta\left(e^{-i \theta}\right)}\right]} e^{i n \theta}\right]+o(1)
$$

where $o(1)$ is in the sense of the $\mathcal{L}_{2, \omega}$ norm. As a last step, we derive the asymptotic behavior for $s_{n}^{(k)}\left(c_{j}\right), 0 \leq k \leq J_{j}, 1 \leq j \leq m$. We consider

$$
S_{n}(x) w_{M}(x)=L_{n+M}(x)+\sum_{j=1}^{2 M} a_{n, j} L_{n+M-j}(x)
$$

where, using the notation introduced in Lemma 9, we denote

$$
g_{j}(x):=\frac{w_{M}(x)}{\left(x-c_{j}\right)^{J_{j}+1}}=\prod_{\substack{i=1 \\ i \neq j}}^{m}\left(x-c_{i}\right)^{J_{i}+1} .
$$

From orthogonality for the Sobolev inner product, for $n>M-1$, we get

$$
\begin{aligned}
0 & =\left\langle g_{j}(x)\left(x-c_{j}\right)^{J_{j}}, S_{n}(x)\right\rangle \\
& =\int_{-1}^{1} \frac{w_{M}(x) S_{n}(x)}{x-c_{j}} d \mu(x)+\left(0, \cdots, 0, J_{j} ! g_{j}\left(c_{j}\right)\right) \mu_{j}\left(\begin{array}{c}
S_{n}\left(c_{j}\right) \\
\vdots \\
S_{n}^{\left(J_{j}\right)}\left(c_{j}\right)
\end{array}\right),
\end{aligned}
$$




$$
\begin{aligned}
& 0=\left\langle g_{j}(x)\left(x-c_{j}\right)^{J_{j}-1}, S_{n}(x)\right\rangle \\
& =\int_{-1}^{1} \frac{w_{M}(x) S_{n}(x)}{\left(x-c_{j}\right)^{2}} d \mu(x) \\
& +\left(0, \cdots, 0,\left(J_{j}-1\right) ! g_{j}\left(c_{j}\right),\left(\begin{array}{c}
J_{j} \\
J_{j}-1
\end{array}\right)\left(J_{j}-1\right) ! g^{\prime}\left(c_{j}\right)\right) \mu_{j}\left(\begin{array}{c}
S_{n}\left(c_{j}\right) \\
\vdots \\
S_{n}^{\left(J_{j}\right)}\left(c_{j}\right)
\end{array}\right), \\
& 0=\left\langle g_{j}(x), S_{n}(x)\right\rangle \\
& =\int_{-1}^{1} \frac{w_{M}(x) S_{n}(x)}{\left(x-c_{j}\right)^{J_{j}+1}} d \mu(x)+\left(g_{j}\left(c_{j}\right), g_{j}^{\prime}\left(c_{j}\right), \cdots, g_{j}^{\left(J_{j}\right)}\left(c_{j}\right)\right) \mu_{j}\left(\begin{array}{c}
S_{n}\left(c_{j}\right) \\
\vdots \\
S_{n}^{\left(J_{j}\right)}\left(c_{j}\right)
\end{array}\right) .
\end{aligned}
$$

Taking into account the matrix $G_{j}$ which appears in the statement of the theorem, we can express, for every $j, 1 \leq j \leq m$, the above equations in matrix form.

$$
G_{j} \mu_{j}\left(\begin{array}{c}
s_{n}\left(c_{j}\right) \\
\vdots \\
s_{n}^{\left(J_{j}\right)}\left(c_{j}\right)
\end{array}\right)=-\gamma_{n}\left(\begin{array}{c}
\int_{-1}^{1} \frac{w_{M}(x) S_{n}(x)}{\left(x-c_{j}\right)} \omega(x) d x \\
\vdots \\
\int_{-1}^{1} \frac{w_{M}(x) S_{n}(x)}{\left(x-c_{j}\right)^{J_{j}+1}} \omega(x) d x
\end{array}\right)
$$

To obtain an asymptotic formula, we must study the asymptotic behavior of the righthand side of (13). Using the change the variable $\frac{1}{2}(z+1 / z)=\lambda$, the asymptotics of $L_{n}$ on $[-1,1]$ as well as $(11)$, we have

$$
\begin{aligned}
\int_{-1}^{1} \frac{w_{M}(x) S_{n}(x)}{\left(x-c_{j}\right)} \omega(x) d x \\
=\left[\int_{-1}^{1} \frac{L_{n+M}(x)}{(x-\lambda)} \omega(x) d x+\sum_{j=1}^{2 M} a_{n, j} \int_{-1}^{1} \frac{L_{n+M-j}(x)}{(x-\lambda)} \omega(x) d x\right]_{\lambda=c_{j}} \\
=\left[\frac{z^{n}}{2^{n}}\left[\left(\frac{z}{2}\right)^{M}+\sum_{j=1}^{2 M} a_{j}\left(\frac{z}{2}\right)^{M-j}\right] \int_{-\pi}^{\pi} \frac{D_{\omega}(z) D_{\omega}(0) d \theta}{(\cos \theta-\lambda)}\right]_{\substack{\lambda=c_{j} \\
z=z_{j}}}[1+o(1)] \\
=\left[\frac{4 \pi}{z-1 / z} D_{\omega}(z) D_{\omega}(0) \frac{z^{n-M}}{2^{n+M}} \prod_{k=1}^{m}\left(z-\frac{1}{z_{k}}\right)^{2\left(J_{k}+1\right)}\right]_{z=z_{j}}[1+o(1)]
\end{aligned}
$$

where we have used

$$
\begin{aligned}
\int_{-1}^{1} \frac{L_{n}(x)}{x-\lambda} \omega(x) d x & =\frac{1}{L_{n}(\lambda)} \int_{-1}^{1} \frac{L_{n}^{2}(x)}{x-\lambda} \omega(x) d x \\
& =\frac{D_{\omega}(0)}{2^{n}} D_{\omega}(z) z^{n} \int_{-\pi}^{\pi} \frac{1}{\left|D_{\omega}\left(e^{i \theta}\right)\right|^{2}} \frac{\omega(\cos \theta)|\sin \theta|}{\cos \theta-\lambda} d \theta[1+o(1)]
\end{aligned}
$$


If we denote by

$$
h_{n}(z)=-\frac{\sqrt{2 \pi}}{2^{M-1}} D_{\omega}(z) \frac{1}{z-1 / z} z^{n-M} \prod_{k=1}^{m} \frac{\left(z z_{k}-1\right)^{2\left(J_{k}+1\right)}}{z_{k}^{J_{k}+1}}
$$

then

$$
-\gamma_{n} \int_{-1}^{1} \frac{w_{M}(x) S_{n}(x)}{\left(x-c_{j}\right)} \omega(x) d x=h_{n}\left(z_{j}\right)[1+o(1)] .
$$

For the other coordinates on the right-hand side of (13), we use again the fact that if $f_{n}=g_{n}[1+o(1)]$ where $f_{n}, g_{n}$ are analytic functions and $g_{n}$ is uniformly bounded, then $f_{n}^{\prime}=g_{n}^{\prime}[1+o(1)]$ and

$$
-\int_{-1}^{1} \frac{w_{M}(x) s_{n}(x)}{(x-\lambda)^{k+1}} \omega(x) d x=\frac{1}{k !} \frac{d^{k}}{d \lambda^{k}}\left[h_{n}(z)\right]_{\lambda=c_{j}}[1+o(1)] .
$$

Taking this into account, we obtain

$$
G_{j} \mu_{j}\left(\begin{array}{c}
s_{n}\left(c_{j}\right) \\
s_{n}^{\prime}\left(c_{j}\right) \\
\vdots \\
s_{n}^{\left(J_{j}\right)}\left(c_{j}\right)
\end{array}\right)=\left(\begin{array}{c}
\left.h_{n}(z)\right|_{\lambda=c_{j}} \\
\left.\frac{d}{d \lambda} h_{n}(z)\right|_{\lambda=c_{j}} \\
\vdots \\
\left.\frac{1}{J_{j} !} \frac{d^{J_{j}}}{d \lambda^{J_{j}}} h_{n}(z)\right|_{\lambda=c_{j}}
\end{array}\right)[1+o(1)]
$$

Remark 1. Notice that on obtaining the strong asymptotics on $[-1,1]$, we have not used the condition $I_{j}-1=J_{j}=N_{j}$. At the points $\left\{c_{j}\right\}, j=1, \ldots, m$, this condition is not necessary either but the derivation of the corresponding formula is quite complicated.

Acknowledgments. We wish to express our gratitude to Professor I. A. Aptekarev for suggesting the main problem of this work and also for valuable comments. We also would like to thank the referees for their careful reading of the manuscript and their suggestions.

The work of the authors was supported by Dirección General de Investigación Científica y Técnica (DGICYT) of Spain under grant PB 93-0228-C02-01.

\section{References}

1. I. A. Aptekarev, F. Marcellán, and G. López, Orthogonal polynomials with respect to a differential operator. Existence and uniqueness, Diff. and Integral Eq. (to appear).

2. I. A. Aptekarev and E. Nikishin, The scattering problem for a discrete Sturm-Liouville operator, Mat. Sb. 121 (1983), 327-358; English transl. in Math. USSR Sb. 49 (1984), 325-355.

3. I. A. Aptekarev and H. Stahl, Asymptotics of Hermite-Padé polynomials, In: Progress in Approximation Theory (Ed. A. Gonchar and E. Saff), Springer-Verlag New York, 1992, pp.127-168.

4. P. Delsarte, Y. V. Genin, and Y. G. Kamp, Orthogonal polynomial matrices on the unit circle, IEEE. Trans Circuits and Systems CAS-25 (1978), 149-160.

5. A. J. Durán and W. Van Assche, Orthogonal matrix polynomials and higher order recurrence relations, Linear Algebra and Applications 219 (1995), 261-280.

6. G. López, F. Marcellán, and W. Van Assche, Relative asymptotics for polynomials orthogonal with respect to a discrete Sobolev inner product, Constructive Approximation 11 (1995), 107-137. 
7. F. Marcellán and A. Ronveaux, Orthogonal polynomials and Sobolev inner products. A bibliography, Facultés Universitaires N. D. de la Paix. Namur., Belgium, 1995.

8. F. M arcellán and W. Van Assche, Relative asymptotics for orthogonal polynomials with a Sobolev inner product, J. Approx. Theory 72 (1993), 193-209.

9. E. Nikishin, The discrete Sturm-Liouville operator and some problems of function theory, Trudy Sem. Petrovsk 10 (1984), 3-77; English transl. in Soviet Math 35 (1987), 2679-2744.

10. J. Nuttal, Asymptotics of diagonal Hermite-Padé polynomials, J. Approx. Theory 42 (1984) 299-386.

11. G. Szegő, Orthogonal Polynomials, Fourth Ed., Amer. Math. Soc. Colloq. Publ., Vol. 23, Amer. Math. Soc., Providence, Rhode Island, 1975.

Departamento de Matemáticas, Escuela Politécnica Superior, Universidad Carlos III, C. Butarque, 15, 28911 Leganés-Madrid, Spain.

E-mail: foulquie@dulcinea.uc3m.es, pacomarc@ing.uc3m.es 